\title{
Optimal Design Based on Dynamic Characteristics and Experimental Implementation of Submersible Electromagnetic Actuators
}

\author{
Baoping Cai ${ }^{1}$-Yonghong Liu ${ }^{1, *}$-Aibaibu Abulimiti1 ${ }^{1}$ Renjie Ji1-Yanzhen Zhang ${ }^{1}-$ Xin Dong 1 -Yuming Zhou ${ }^{2}$ \\ ${ }^{1}$ China University of Petroleum, College of Mechanical and Electronic Engineering, China \\ 2 China National Petroleum Corporation, Bureau of Geophysical Prospecting, China
}

An optimization procedure based on the dynamic characteristics of a submersible electromagnetic actuator constrained in a specific volume has been developed. In order to achieve the minimum response time and low vibration when the actuator is opened, five radial dimensions are optimized when the main limiting quantity is the maximum allowed temperature. Three models, including a thermal model, an electromagnetic model, and a mechanical model are constructed, and optimization calculation is performed by using Matlab/Simulink software. According to the optimization results, an actuator is manufactured and dynamic experiments are performed. The results show substantial agreement between experimental response times and simulated response times. In order to investigate the vibration when the actuator is opened and closed, vibration experiments of the actuator are also performed. The results show that the measured vertical and horizontal accelerations are near the calculated acceleration of the plunger. Both of the experiments show that the optimization procedure is sufficiently accurate, and the optimal submersible electromagnetic actuator is sufficiently secure to be used in subsea BOP stacks.

Keywords: submersible electromagnetic actuator, optimal design, magnetic equivalent circuit, dynamic characteristics

\section{INTRODUCTION}

Hundreds of submersible control valves are used in a subsea blowout preventer (BOP) stack, which is located in $3000 \mathrm{~m}$ ultra-deep water region. The control valve is an electro-hydraulic device with two stages. The pilot stage, which is a submersible electromagnetic actuator (SEMA), provides a hydraulic pilot signal to operate a main stage. The main stage is a sub-platemounted (SPM) hydraulic valve, which controls the hydraulic pressure to operate the subsea BOP stack functions [1] and [2]. By minimizing response times, especially the opening time of SEMA, the performance of subsea BOP can be improved significantly. High velocity impacts experienced by the moving plunger of the SEMA create frequent vibrations of the SEMA and SPM valve, which could lead to valve seal failure. Therefore, the velocity impacts and vibrations should be sufficiently low [3] and [4]. Empirically, the vibration acceleration of the SEMA should be lower than $1 \times 10^{-3} \mathrm{~m} / \mathrm{s}^{2}$. Additionally, the size and weight savings of the submersible control valves are of considerable significance in reducing the size of the subsea BOP stack. Due to the complexity of design parameters, the actuators require an optimal design.

In recent years, various analytical, semi-analytical and numerical methods have been presented in order to improve the performance of electromagnetic devices. Moses et al. [5] studied the linear electromagnetic actuators using finite element analysis (FEA) method in accelerating the design process and improving the final design. Wu et al. [6] studied an electromagnetic fast linear actuator, using the FEA method. Yatchev et al. [7] optimized an axisymmetric linear electromagnetic valve actuator, using the FEA method. The method gives a precise determination of the electromagnetic device performance but requires a large amount of computation and time reduction when the method is used for optimization. The FEA method is also problematic for other applications, such as dynamic simulation; therefore, some solutions including opening and closing times could not be obtained. Encica et al. [8] and [9] optimized the electromagnetic actuators using a space-mapping method, and Markovic et al. [10] and [11] analysed an electromechanical actuator, using the SchwarzChristoffel mapping method. The two methods accelerate the optimization processes by exploiting simplified models; however, they cannot easily solve the dynamic characteristics of electromagnetic devices using Matlab/Simulink. Chung and Gweon [12] developed an electromagnetic linear actuator for a mass flow controller, using magnetic equivalent circuit (MEC) method. Cai et al. [13] optimized the submersible solenoid valves for subsea blowout preventers, using MEC method. Batdorff and Lumkes [14] studied an axisymmetric electromagnetic actuator using high-fidelity MEC methods. The method may have some error when predicting electromagnetic force and magnetic flux; however, it is excellent when they are used to optimize and design electromagnetic devices. The dynamic characteristics could be also 
investigated using the methods with Matlab/Simulink environments [15] to [17].

In this paper, an MEC-based optimization design procedure for SEMA, constrained in a specific small volume, is proposed. The optimal design aims to minimize response time, especially the opening time when the main limiting quantity is the maximum allowed temperature. According to the optimization results, a SEMA is manufactured, and its dynamic characteristics are investigated experimentally in order to verify the proposed optimization procedure.

\section{MATHEMATICAL MODEL}

The SEMA is designed as a solenoid-operated switching spring return actuator, which is essentially composed of a cover, a spring, a spring pocket, a coil, a coil bobbin, a plunger, a plunger sleeve, and a magnetic ring, as shown in Fig. 1. The plunger-type structure is intended to be produced in a small size [18]. When the coil is energized by DC voltage, the plunger of the SEMA retracts upward and extends downwards by releasing the stored energy from the spring [13] and [19].

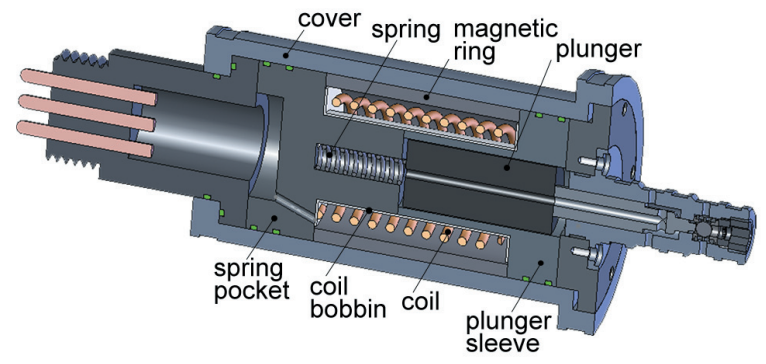

Fig. 1. Schematic diagrams of the SEMA [13]

The cover of the actuator is made of AISI $316 \mathrm{~L}$ austenitic stainless steel due to its high corrosion resistance to seawater, high strength, and high durability [20] and [21]. However, AISI 316L stainless steel is nonmagnetic. Therefore, four components (plunger, spring pocket, plunger sleeve, and magnetic ring) are used to form a magnetic circuit. The plunger is made of iron, which is a ferromagnetic material. The three other components are made of AISI 440C martensitic stainless steel, which is strongly magnetic but has lower corrosion resistance to seawater than AISI 316L austenitic stainless steel [22]. All of the gaps within the SEMA, e.g. the gap between the coil and the magnetic ring, are filled with conduction oil in order to transfer heat power and prevent high-pressure seawater from crushing the actuator. The coil conducts the current that provides magnetic flux, and it consists of numerous turns and layers of conducting copper wire, insulation and bonding material. The geometry of the SEMA is shown in Fig. 2 [13].

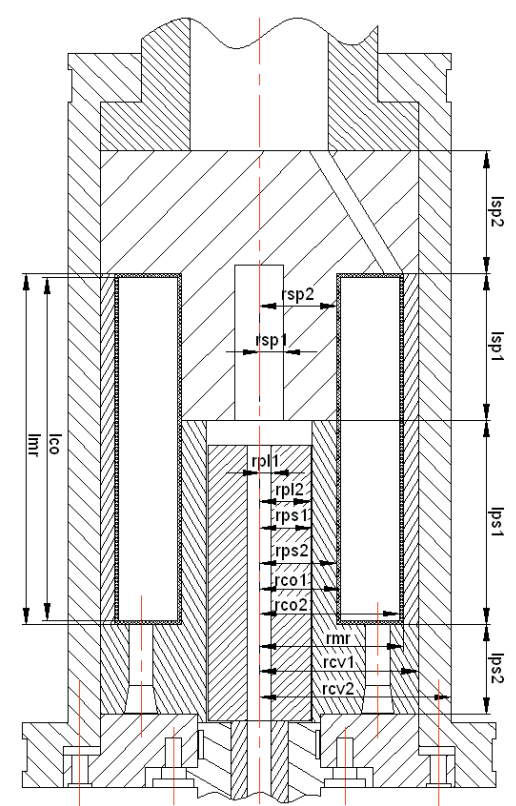

Fig. 2. Geometry of the SEMA [13]

\subsection{Thermal Model}

The electrical circuit of an electromagnetic actuator supplies current to the coils. This current flows through wires and produces heat due to the wellknown Joule effect. The magnetic circuit provides the flux and the force, also producing heat due to the magnetic losses in the magnetic circuit [23]. Although some magnetic (demagnetization, saturation and magnetization hysteresis) and mechanical (friction and mechanical stress) effects are significant in electromagnetic actuators, the main limiting quantity considered in this study is the maximum allowed temperature of the SEMA in seawater, which is similar to the precondition of the solenoid actuator designed in [24]. Therefore, to minimize the response time for a specific small volume actuator, a study of the heat transfer phenomenon is to be done. A series of expressions has been developed in order to maintain the temperature of the actuator under the safety threshold.

For simplicity of design, this study assumes that the coil bobbin is adiabatic, and the maximum temperature is in the centre of the coil; hence, the heat power, produced in the coil due to the energizing current, transfers from the coil to seawater via the conduction oil, the magnetic ring, and the cover of 
the actuator. For the sake of safety and simplicity, continuous operation ( $100 \%$ duty cycle) has been assumed [25]. The steady-state heating balance can be expressed as follows:

$$
i_{\max }^{2} R=\frac{T_{c o}-T_{s e a}}{\zeta_{c o}+\zeta_{o i l}+\zeta_{m r}+\zeta_{c v}},
$$

where $i_{\max }$ is the maximum allowed current in the coil, $R$ is the resistance of the coil, and it is associated with the dimension, resistivity, resistivity temperature coefficient and number of coil turns of the SEMA [13]. $T_{c o}$ and $T_{\text {sea }}$ are the temperature of the coil and seawater, respectively, and $\zeta_{c o}, \zeta_{o i l}, \zeta_{m r}$ and $\zeta_{c v}$ are the thermal resistances of the coil, the conduction oil, the magnetic ring, and the cover, respectively, and they are associated with the dimension, conduction coefficients of the coil, and convection coefficient of the conduction oil [13].

The current factor $\kappa$ is defined as the ratio of the rated current to the maximum current. Therefore, the rated current can be derived from Eq. (3) and written as follows:

$$
i_{e}=\kappa i_{\max } .
$$

By applying the Ohm law, the assigned coil rated voltage can be written as follows:

$$
U_{e}=i_{e} R
$$

\subsection{Electromagnetic Model}

The electromagnetic model of the SEMA consists of an electrical circuit and a magnetic circuit. The electrical circuit is the actual coil, and the magnetic circuit consists of the plunger, the plunger sleeve, the spring pocket, and the magnetic ring. By applying Kirchhoff's voltage law, the magnetic and electrical circuits yields:

$$
U_{e}=i(t) R+N \frac{d \varphi(t)}{d t},
$$

where $U_{e}$ is the input coil rated voltage, $i(t)$ is the electrical current, $R$ is the resistance of the coil, $N$ is the number of turns, and $\phi(t)$ is the total magnetic flux.

According to the magnetic equivalent circuit of the SEMA, The electromotive force can be expressed as follows:

$$
i(t) N=\varphi(t) R_{t m},
$$

where $R_{t m}$ is the total magnetic reluctance.
The relationship of $i(t), N, \phi_{1}(t), \phi_{2}(t)$ and $R$ can be obtained from an analogy of the electric circuit and the magnetic circuit and can be expressed as follows:

$$
\begin{aligned}
\frac{N i(t)}{\varphi_{1}(t)+\varphi_{2}(t)} & =\frac{\left(R_{g a}+R_{p l}+R_{g p}\right) R_{p s 1}}{R_{g a}+R_{p l}+R_{g p}+R_{p s 1}}+ \\
& +R_{s p 1}+R_{s p 2}+R_{m r}+R_{p s 2}, \\
\frac{\varphi_{1}(t)}{\varphi_{2}(t)} & =\frac{R_{p s 1}}{R_{g a}+R_{p l}+R_{g p}},
\end{aligned}
$$

where $\phi_{1}(t)$ and $\phi_{2}(t)$ are the magnetic flux flowing inside the plunger and the upper plunger sleeve, respectively. $R_{g a}, R_{p l}, R_{g p}, R_{p s 1}, R_{p s 2}, R_{s p 1}, R_{s p 2}$ and $R_{m r}$ are the magnetic reluctances of the magnetic flux paths of working gap, plunger, gap between the plunger and plunger sleeve, upper plunger sleeve, lower plunger sleeve, lower spring pocket, upper spring pocket and magnetic ring (see Figs. 1 and 2), respectively.

For the ferromagnetic parts, consisting of the plunger, the plunger sleeve, the spring pocket, and the magnetic ring, the empirical curve-fit for the B-H curve is used and expressed as follows [26]:

$$
\begin{gathered}
B_{p l}=\mu_{p l} H_{p l}=\frac{C_{1} H_{p l}}{1+C_{2} H_{p l}}+C_{3} H_{p l}, \\
B_{i}=\mu_{i} H_{i}=\frac{C_{4} H_{i}}{1+C_{5} H_{i}}+C_{6} H_{i},
\end{gathered}
$$

where $i$ denotes $p s 1, p s 2, s p 1, s p 2$, and $m r, B$ is the magnetic flux density, $H$ is the magnetic field intensity, $C_{1}, C_{2}, C_{3}, C_{4}, C_{5}$, and $C_{6}$ are regression parameters, and $\mu_{p l}, \mu_{i}$ are the magnetic permeability of iron and AISI $440 \mathrm{C}$ stainless steel respectively, which are both variable.

Assuming uniform flux density across the crosssectional area of the plunger, the plunger sleeve, the spring pocket, the magnetic ring, and the two gaps, the magnetic flux density can be expressed as follows:

$$
\begin{gathered}
B_{p l}=\frac{\varphi_{1}(t)}{A_{p l}}, \\
B_{p s 1}=\frac{\varphi_{2}(t)}{A_{p s 1}}, \\
B_{i}=\frac{\varphi(t)}{A_{i}},
\end{gathered}
$$

where $i$ denotes $p s 2, s p 1, s p 2$, and $m r$. 
Substituting Eq. (10) into Eq. (8), and Eqs. (11) and (12) into Eq. (9), the magnetic permeability of $\mu_{p 1}$, $\mu_{p s 1}$ and $\mu_{i}$ can be expressed as functions of $\phi_{1}(t), \phi_{2}(t)$ and $\phi(t)$ as follows:

$$
\begin{aligned}
\mu_{p l}= & f_{p l}\left(\varphi_{1}(t)\right)=\frac{\left(C_{1}+C_{3}\right) A_{p l}-C_{2} \varphi_{1}(t)}{2 A_{p l}}+ \\
& +\frac{\left\{\left[\left(C_{1}+C_{3}\right) A_{p l}-C_{2} \varphi_{1}(t)\right]^{2}+4 \varphi_{1}(t) C_{2} C_{3} A_{p l}\right\}^{1 / 2}}{2 A_{p l}}, \\
\mu_{p s 1}= & f_{p s 1}\left(\varphi_{2}(t)\right)=\frac{\left(C_{4}+C_{6}\right) A_{p s 1}-C_{5} \varphi_{2}(t)}{2 A_{p s 1}}+ \\
& +\frac{\left\{\left[\left(C_{4}+C_{6}\right) A_{p s 1}-C_{5} \varphi_{2}(t)\right]^{2}+4 \varphi_{2}(t) C_{5} C_{6} A_{p s 1}\right\}^{1 / 2}}{2 A_{p s 1}}, \\
\mu_{i}= & f_{i}(\varphi(t))=\frac{\left(C_{4}+C_{6}\right) A_{i}-C_{5} \varphi(t)}{2 A_{i}}+ \\
& +\frac{\left\{\left[\left(C_{4}+C_{6}\right) A_{i}-C_{5} \varphi(t)\right]^{2}+4 \varphi(t) C_{5} C_{6} A_{i}\right\}^{1 / 2}}{2 A_{i}},
\end{aligned}
$$

where $i$ denotes $p s 2, s p 1, s p 2$, and $m r$.

Therefore, the relationship of parameters above can be rewritten as follows:

$$
\left\{\begin{array}{l}
\frac{N i_{e}}{\varphi_{1}(t)+\varphi_{2}(t)}=\frac{\left[\frac{L_{g a}}{\mu_{0} A_{g a}}+\frac{L_{p l}}{f_{p l}\left(\varphi_{1}(t)\right) A_{p l}}+\frac{L_{g p}}{\mu_{0} A_{g p}}\right] \frac{L_{p s 1}}{\frac{L}{g a}_{0} A_{g a}}+\frac{\left.L_{p l}(t)\right) A_{p s 1}}{f_{p l}\left(\varphi_{1}(t)\right) A_{p l}}+\frac{L_{g p}}{\mu_{0} A_{g p}}+\frac{L_{p s 1}}{f_{p s 1}\left(\varphi_{2}(t)\right) A_{p s 1}}}{+\frac{L_{s p 1}}{f_{s p 1}(\varphi(t)) A_{s p 1}}+\frac{L_{s p 2}}{f_{s p 2}(\varphi(t)) A_{s p 2}}+\frac{L_{p r}}{f_{m r}(\varphi(t)) A_{m r}}+\frac{L_{p s 2}}{f_{p s 2}(\varphi(t)) A_{p s 2}}} \\
\frac{\varphi_{1}}{\varphi_{2}}=\frac{\frac{L_{p s 1}}{f_{p s 1}\left(\varphi_{2}(t)\right) A_{p s 1}}}{\frac{L_{g a}}{\mu_{0} A_{g a}}+\frac{L_{p l}}{f_{p l}\left(\varphi_{1}(t)\right) A_{p l}}+\frac{L_{g p}}{\mu_{0} A_{g p}}}
\end{array} .\right.
$$

As shown in Eq. (16), the system of equations, consisted of two unknown quantities $\phi_{1}(t)$ and $\phi_{2}(t)$, can be solved using an iterative procedure.

The electromagnetic force can be expressed as:

$$
F_{\text {mag }}=\frac{\varphi_{1}^{2}(t)}{2 \mu_{0} A_{g a}} .
$$

The magnetic flux $\phi_{1}(t)$ depends on the variation of the length of the working gap according to Eq. (16). Therefore, electromagnetic force also depends on the length of the working gap.

\subsection{Mechanical Model}

For optimal design purposes, a simpler second-order, one-degree of freedom model has been adopted. The mechanical model consists of a mass, spring and damper under the effect of magnetic and pressure forces, which can be represented by Newton's second law as:

$$
m \frac{d^{2} x(t)}{d t^{2}}+b \frac{d x(t)}{d t}+k(\delta+x(t))=F_{m a g}(t)+F_{p r e},
$$

where $x$ is the displacement of the plunger $(x=0$ when the actuator is opened, and $x=2.7 \times 10^{-3} \mathrm{~m}$ when it is closed), $m$ is the total movable mass, $b$ is the lumped damping coefficient, $k$ is the effective spring coefficient, $\delta$ is the spring pre-tension, $F_{\text {mag }}$ are the electromagnetic force, and $F_{\text {pre }}$ are the pressure force. During the optimal design, the pressure force is considered as constant.

\section{OPTIMIZATION AND DISCUSSION}

The SEMA is constrained in a specific small volume with constant length and diameter. The objective is to optimize the designs of the individual components in order to achieve the minimum response time when the actuator is opened. Five radial dimensions including the thickness of the plunger $\left(w_{p l}\right)$, the thickness of the upper plunger sleeve $\left(w_{p s}\right)$, the thickness of the coil $\left(w_{c o}\right)$, the thickness of the magnetic ring $\left(w_{m r}\right)$, and the gap between the plunger and plunger sleeve $\left(w_{g p}\right)$ are optimized when the other radial dimensions are constant.

The geometric optimization of SEMA is performed by using Matlab/Simulink software. The Simulink model consists of three main parts: the thermal subsystem, the electromagnetic subsystem, and the mechanical subsystem, as shown in Fig. 3. The thermal subsystem solves the number of turns, the resistance of the coil, and the rated voltage based on the five input radial dimensions when the initial main parameters are set as follows: the maximum allowed temperature of the SEMA is $120^{\circ} \mathrm{C}$ because the synthetic enamelled copper wire will be burned down and short circuit when the temperature is higher than $120^{\circ} \mathrm{C}$, and the ambient seawater temperature is $4{ }^{\circ} \mathrm{C}$. The three parameters solved by the thermal subsystem with the displacement of the plunger fed back from the mechanical subsystem are then input to the electromagnetic subsystem, and the electrical current and electromagnetic force can be solved. Using the electromagnetic force, the displacement, velocity and acceleration of the plunger are solved in the mechanical subsystem. 


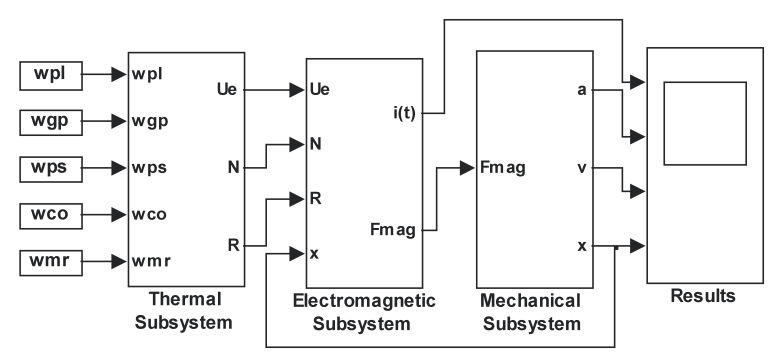

Fig. 3. Simulink model of the SEMA for dynamic characteristics
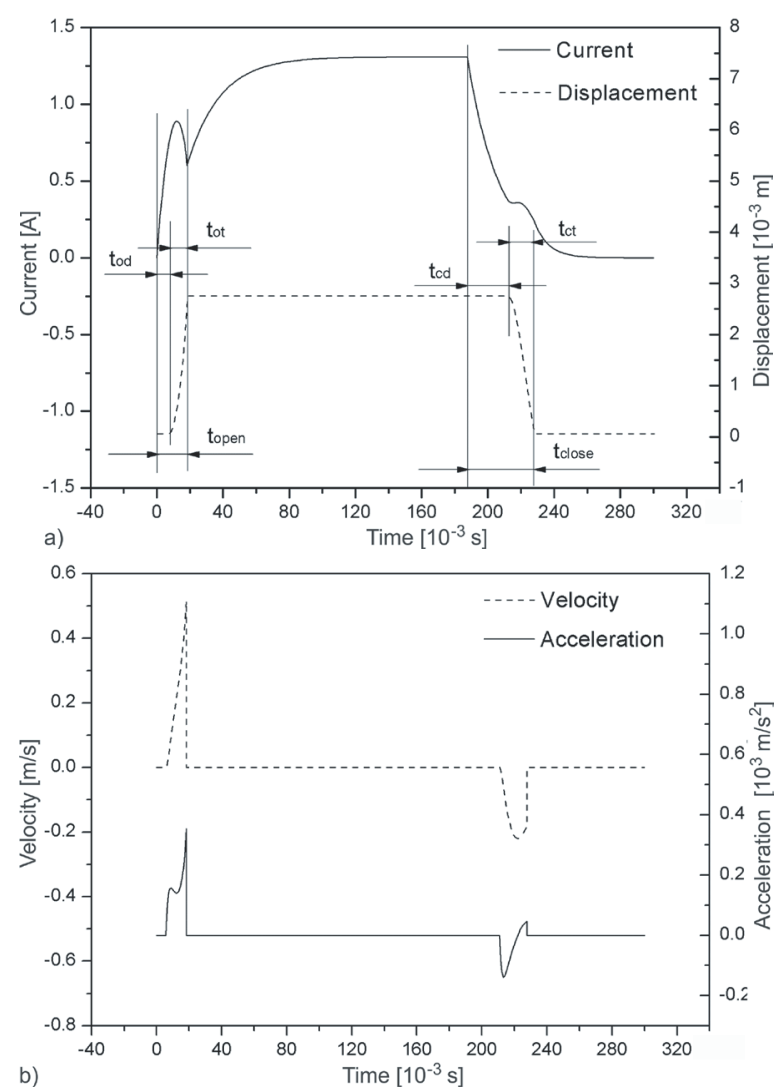

Fig. 4. Simulated dynamic characteristics of the SEMA; a) current of the coil and displacement of the plunger, and b) velocity and acceleration of the plunger

The simulated dynamic characteristics, including current of the coil, displacement, velocity and acceleration of the plunger for the SEMA with certain radial dimensions are shown in Fig. 4. When the coil is energized, the current increases rapidly until the sum of magnetic force and pressure force exceeds the spring force. This time period is considered to be the plunger delay time $\left(t_{o d}\right)$. As the plunger starts to move, the inductance of the coil starts to increase due to the decreasing working gap. Therefore, the effective time constant increases, and the current of the coil decreases until the plunger reaches its final position. This time period is considered as plunger travel time $\left(t_{o t}\right)$. Therefore, the total opening time of the actuator $\left(t_{\text {open }}\right)$ is the sum of the plunger delay time and plunger travel time. Similarly, the total closing time of the actuator $\left(t_{\text {close }}\right)$ is the sum of the plunger delay time $\left(t_{c d}\right)$ and plunger travel time $\left(t_{c t}\right)$ when the coil is deenergized. It is governed by the force balance between the pressure force, the spring force, and the magnetic holding force. The current and holding force decay in the de-energized magnet are not instantaneous, but they follow a transient state. This retards the beginning of the motion of the plunger depending on the level of holding current [27].

By inputting the combination of different values of $w_{p s}$ and $w_{g p}, w_{p l}$ and $w_{m r}$, and wmr and wco shown in Fig. 3, the response time can be obtained from the dynamic characteristic curves shown in Fig. 4. The response time including opening time and closing time as functions of the five radial dimensions is shown in Fig. 5. As shown in Figs. 5a and b, when the initial two dimensions are set as $w_{p l}=5 \times 10^{-3} \mathrm{~m}$, and $w_{m r}=2 \times 10^{-3} \mathrm{~m}$, as the thickness of the upper plunger sleeve $\left(w_{p s}\right)$ and the gap between the plunger and the plunger sleeve $\left(w_{g p}\right)$ decreases, the opening time increases and the closing time decreases. The smaller the magnetic flux flows to the upper plunger sleeve, the bigger it flows to plunger, which is analogous to current of a parallel electric circuit. A big magnetic flux produces a big magnetic force and a short opening time. The big magnetic flux also produces big holding current and subsequent long closing time. For the SEMA used in subsea BOP stack, closing time is secondary to opening time. In order to maximize the opening time, the thickness of the upper plunger sleeve has to be minimized. However, if the thickness is too small, its strength is significantly weakened. Therefore, a suitable thick upper plunger sleeve should be used. Similarly, the small gap between the plunger and plunger sleeve produces small magnetic reluctance, a big magnetic flux, a big magnetic force and a short opening time. However, the small gap also produces major friction between the plunger and the plunger sleeve. Therefore, based on the overall consideration of various factors including opening time, closing time, strength and friction, the two design dimensions are set as $w_{p s}=1.8 \times 10^{-3} \mathrm{~m}$ and $w_{g p}=0.2 \times 10^{-3} \mathrm{~m}$.

When the two dimensions are set as $w_{p s}=$ $1.8 \times 10^{-3} \mathrm{~m}$ and $w_{g p}=0.2 \times 10^{-3} \mathrm{~m}$, the opening time and closing time as functions of the thickness of the plunger (wpl), the magnetic ring (wmr) and the coil (wco) are shown in Figs. 5c to f. The three dimensions are dependent on each other due to the fact that the sum of the three values is a constant when the two 
other are constant. With increasing $w_{p l}$ and $w_{m r}$, the opening time increases and the closing time decreases. When $w_{p l}$ is $6.0 \times 10^{-3} \mathrm{~m}$, and $w_{m r}$ is $3.0 \times 10^{-3} \mathrm{~m}$, the opening time reaches the minimum of around $15 \times 10^{-3}$ $\mathrm{ms}$, and the closing time is about $36 \times 10^{-3} \mathrm{~s}$. Therefore, the optimal resolution of the SEMA is $w_{p l}=6.0^{\circ} \times 10^{-3}$ $\mathrm{m}, w_{c o}=6.0 \times 10^{-3} \mathrm{~m}$, and $w_{m r}=3.0 \times 10^{-3} \mathrm{~m}, w_{p s}=$ $1.8 \times 10^{-3} \mathrm{~m}, w_{g p}=0.2 \times 10^{-3} \mathrm{~m}$. For the optimal SEMA, the rated voltage $\left(U_{e}\right)$ is $16 \mathrm{~V}$ and the rated current $\left(i_{e}\right)$ is $1.3 \mathrm{~A}$.
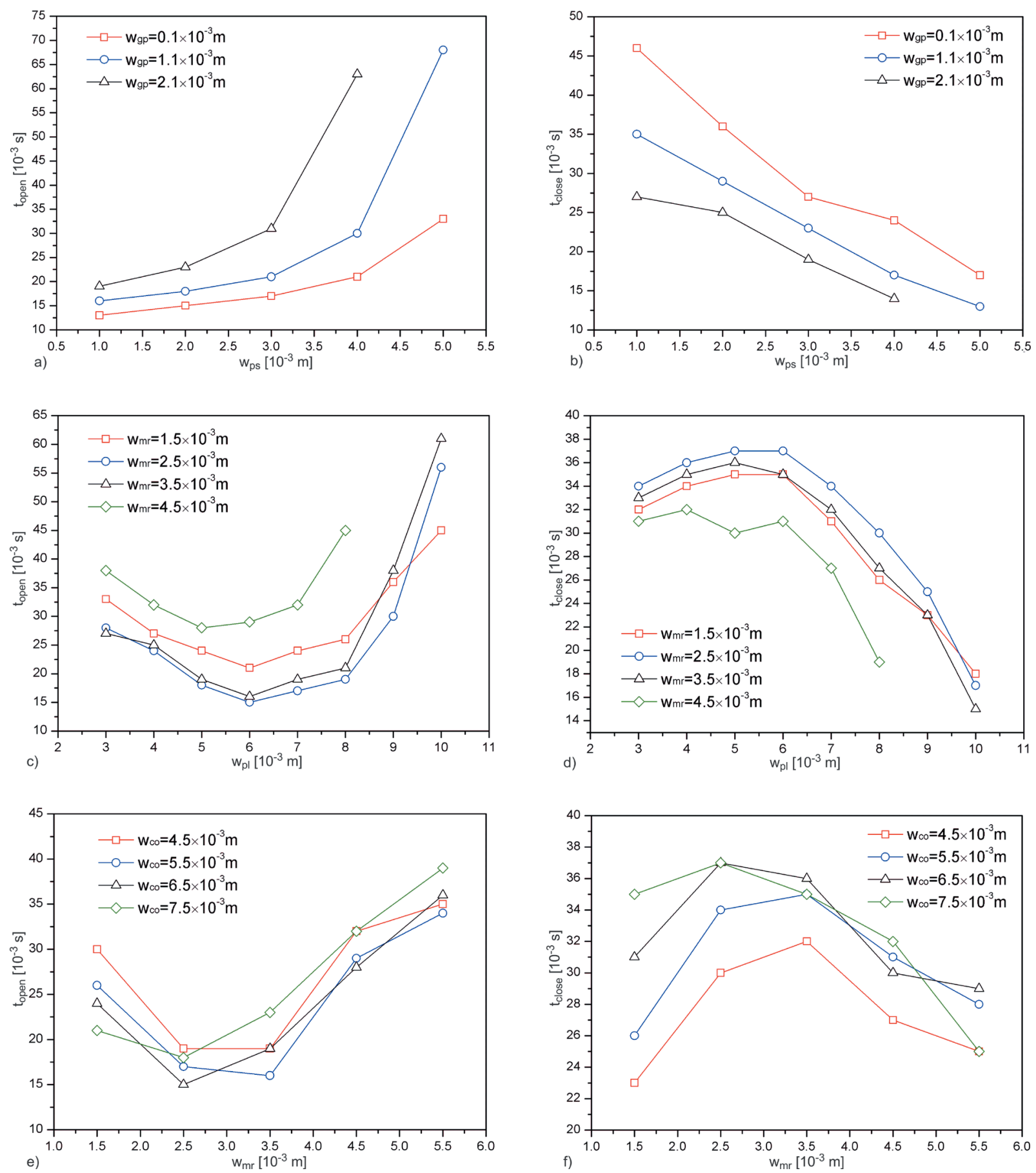

Fig. 5. Optimization results of the SMEA: a) Opening time as a function of $w_{p s}$ and $w_{g p}$, b) Closing time as a function of $w_{p s}$ and $w_{g p}$, c) Opening time as a function of $w_{p l}$ and $\left.w_{m r}, d\right)$ Closing time as a function of $w_{p l}$ and $w_{m r}$, e) Opening time as a function of $w_{m r}$ and $w_{c 0}$, f) Closing time as a function of $w_{m r}$ and $w_{c o}$ 


\section{EXPERIMENTS AND DISCUSSION}

According to the optimization results, a SEMA was manufactured and its dynamic experiment was also performed, as shown in Fig. 6. The DC power was supplied by a switching power supply (Zhaoxin, RXN$3020 \mathrm{D}$, China), which was set to $16 \mathrm{~V}$. The current of the coil was measured by a current transducer (LEM, LTS 6-NP, Switzerland). Because it was very difficult to measure the displacement of the plunger with the hydraulic pressure, the hydraulic pressure was replaced by a long spring under the plunger, which produced a nearly constant force. The displacement was measured with an eddy current displacement sensor (Jingxin, JX70-04-B-M16*1-75-03K, China), which was fixed in an extension rod connected to the plunger. The signals of current and displacement were acquired synchronously by an oscillograph and processed with a computer.

In order to acquire accurate vibration acceleration signals, the moment the plunger reached its
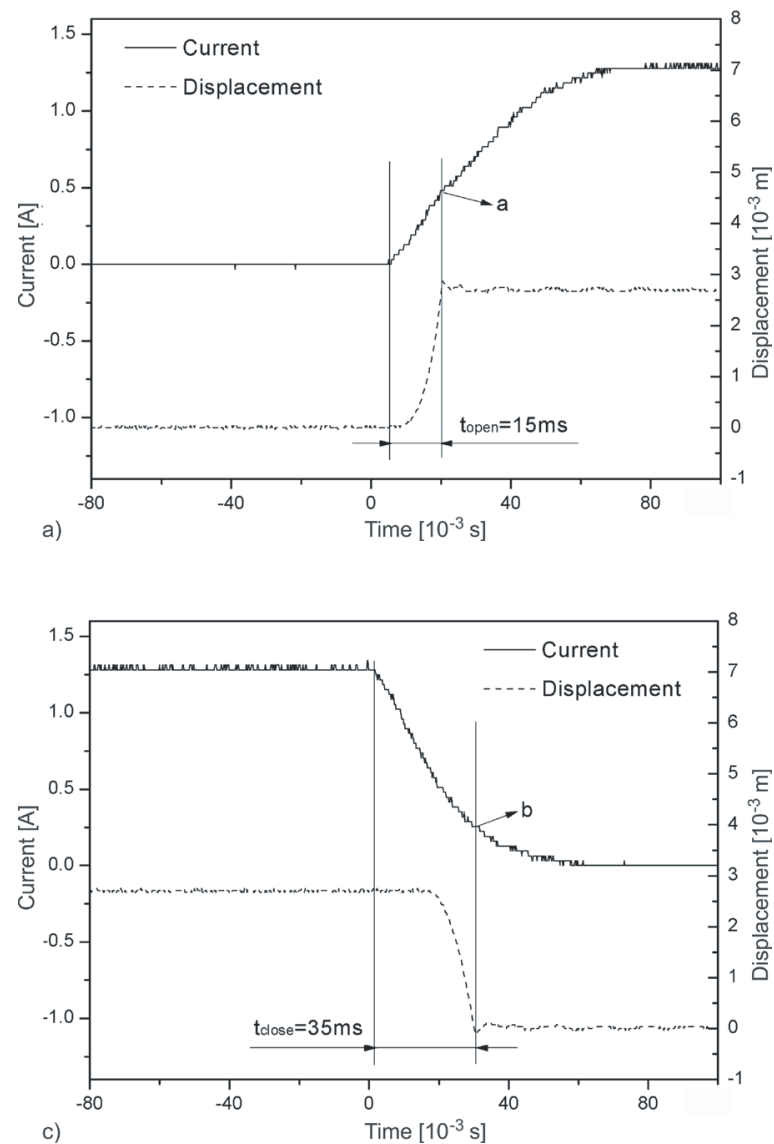

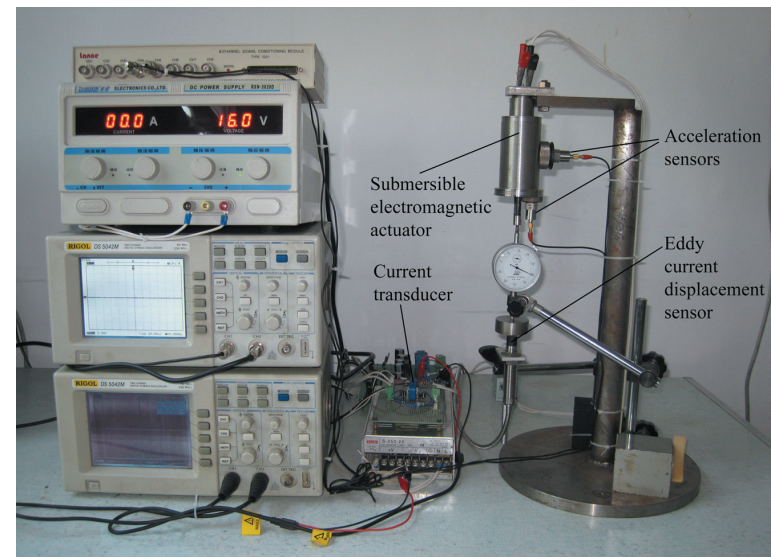

Fig. 6. Schematic of the dynamic experiments of the SEMA

final positions, vertical and horizontal vibration experiments of the SEMA were also performed. Two acceleration sensors (Lance, LC0152, China) were fixed on the top cover (or bottom cover) and cylinder cover of the optimal actuator. Because the sensors
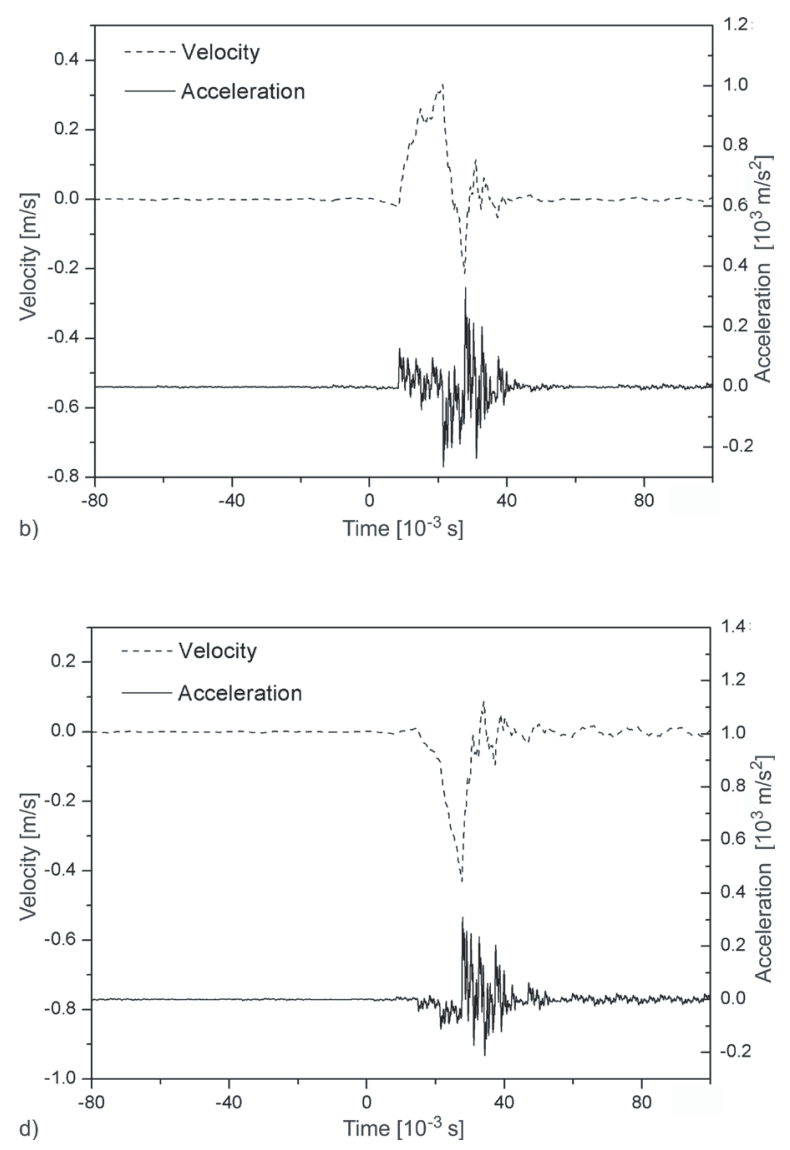

Fig. 7. Experimental dynamic characteristics of the SEMA; a) measured current and displacement and

b) calculated velocity and acceleration when the actuator is opened; c) Measured current and displacement, and d) calculated velocity and acceleration when the actuator is closed 

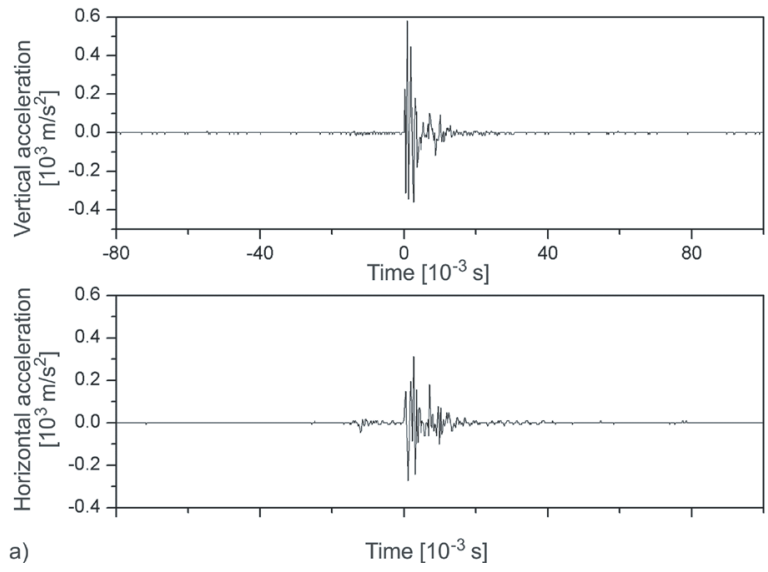

Fig. 8. The vertical and horizontal vibration accelerations of the SEMA when it is; a) opened and b) closed

were not fixed on the plunger, the acceleration when the plunger was moving could not be measured well. However, the rebound problem when the plunger hit the spring pocket, and the bottom cover was represented accurately. The signals of vertical and horizontal vibration acceleration were acquired synchronously by another oscillograph and also processed by the computer.

The experimental dynamic characteristics of the SEMA are shown in Fig. 7. Although it is not obvious that the current decreases to its local minimum when the plunger reaches its final positions (Point a and Point $\mathrm{b}$ shown in Figs. 7a and $\mathrm{c}$ ), the opening time and closing time can also be read clearly. From the measured current and displacement, it can be seen that the opening time and closing time are $15 \times 10^{-3} \mathrm{~s}$ and $35 \times 10^{-3} \mathrm{~s}$, respectively, which shows good agreement with the optimization results. Figs. $7 \mathrm{~b}$ and $\mathrm{d}$ are the calculated velocity and acceleration according to the displacement of the plunger. They clearly differ from the simulated velocity and acceleration shown in Fig. $4 \mathrm{~b}$. This is because during the simulation, the rebound problem of impact when the plunger hits the spring pocket and the bottom cover of the actuator is not considered. In addition, the pressure force is replaced by spring force, which also causes some errors.

The vertical and horizontal vibration accelerations when the SEMA is opened and closed are shown in Fig. 8. After comparing Figs. 7b and 8a, it can be seen that when the actuator is opened, the measured maximum vertical acceleration of $0.6 \times 10^{3}$ $\mathrm{m} / \mathrm{s}^{2}$ is higher than the calculated acceleration of the plunger of $0.4 \times 10^{3} \mathrm{~m} / \mathrm{s}^{2}$, and the measured maximum horizontal acceleration of $0.3 \times 10^{3} \mathrm{~m} / \mathrm{s}^{2}$ is slightly lower than the calculated one. Similarly, as shown in Fig. 7d and Fig. 8b, when the actuator is closed, the measured maximum vertical acceleration of
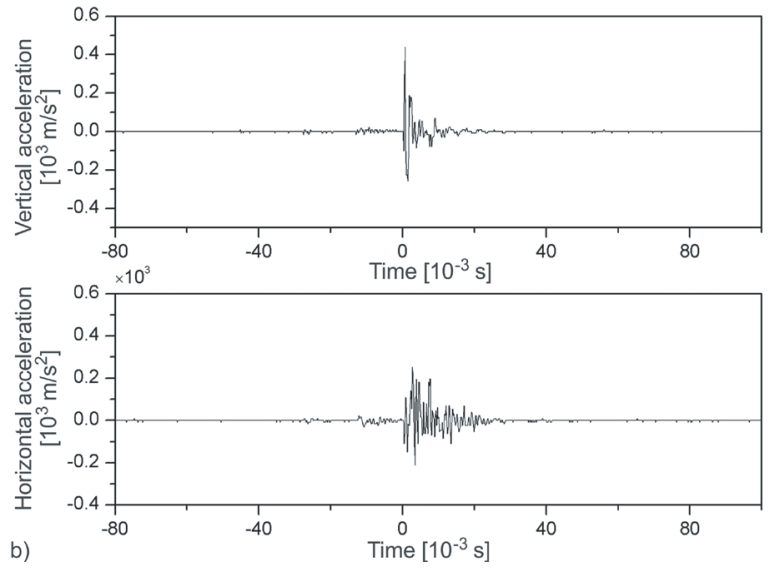

$0.4 \times 10^{3} \mathrm{~m} / \mathrm{s}^{2}$ is higher than the calculated acceleration of the plunger of $0.3 \times 10^{3} \mathrm{~m} / \mathrm{s}^{2}$, and the measured maximum horizontal acceleration of $0.25 \times 10^{3} \mathrm{~m} / \mathrm{s}^{2}$ is slightly lower than the calculated one. This is mainly because it may cause some errors when calculating the second-order derivative of the displacement, the calculated accelerations are lower that the measured ones. Although the measured vertical and horizontal vibration accelerations are higher slightly, they are lower than the empirical maximum value of $1 \times 10^{3}$ $\mathrm{m} / \mathrm{s}^{2}$; therefore, the vibrations cannot affect the sealing of the SEMA.

\section{CONCLUSIONS}

An optimization procedure based on dynamic characteristics of the SEMA constrained in a specific volume has been developed. The objective is to optimize the designs of the individual components in order to achieve the minimum response time, especially the opening time, when the actuator is opened. Three models including thermal model, electromagnetic model, and mechanical model have been built, and five radial dimension including $w_{p l}$, $w_{p s}, w_{m r}, w_{g p}$ and $w_{g p}$ have been optimized by using Matlab/Simulink when the other radial dimensions are constant. The optimal parameters of the SEMA are $w_{p s}=1.8 \times 10^{-3} \mathrm{~m}, w_{g p}=0.2 \times 10^{-3} \mathrm{~m}, w_{p l}=6 \times 10^{-3}$ $\mathrm{m}, w_{c o}=6 \times 10^{-3} \mathrm{~m}$, and $w_{m r}=3 \times 10^{-3} \mathrm{~m}$. An optimal SEMA are manufactured, and a dynamic experiment was performed on it. The results show good agreement between the experimental response time and simulated response time. A vibration experiment of the SEMA was also performed, which shows that the measured vertical and horizontal accelerations are near the calculated acceleration of the plunger. The experiments show that the optimization procedure is 
accurate, and the optimal SEMA is sufficiently secure that it can be used to control subsea BOP stacks.

\section{ACKNOWLEDGEMENTS}

The authors wish to acknowledge the financial support of the National High-Technology Research and Development Program of China (No. 2013AA09A220), National Natural Science Foundation of China (No. 51205411), Program for Changjiang Scholars and Innovative Research Team in University (IRT1086), Taishan Scholar Project of Shandong Province (TS20110823), Science and Technology Development Project of Shandong Province (2011GHY11520) and the Fundamental Research Funds for the Central Universities (No. 13CX02077A).

\section{REFERENCES}

[1] API Specification 16D (2004). Specification for Control Systems for Drilling Well Control Equipment and Control Systems for Diverter Equipment, American Petroleum Institute, Washington.

[2] Turner, P.A. (2001). Material compatibility in directional control valve designs. 2001 Offshore Technology Conference, OTC13233, DOI:10.4043/13233-MS.

[3] Peterson, K.S., Stefanopoulou, A.G. (2004). Extremum seeking control for soft landing of an electromechanical valve actuator. Automatica, vol. 40, no. 6, p. 10631069, DOI:10.1016/j.automatica.2004.01.027.

[4] Eyabi, P., Washington, G. (2006). Modeling and sensorless control of an electromagnetic valve actuator. Mechatronics, vol. 16, no. 3-4, p. 159-175, DOI:10.1016/j.mechatronics.2005.11.008.

[5] Moses, A.J, Al-Naemi, F., Hall, J. (2003). Designing and prototyping for production. Practical applications of electromagnetic modeling. Journal of Magnetism and Magnetic Materials, vol. 254-255, p. 228-233, DOI:10.1016/S0304-8853(02)00963-0.

[6] Wu, D., Xie, X.D., Zhou, S.Y. (2010). Design of a normal stress electromagnetic fast linear actuator. IEEE Transactions on Magnetics, vol. 46, no. 4, p. 10071014, DOI:10.1109/TMAG.2009.2036606.

[7] Yatchev, I., Gueorgiev, V., Hinov, K. (2009). Optimization of an axisymmetric linear electromagnetic valve actuator. COMPEL - The International Journal for Computation and Mathematics in Electrical and Electronic Engineering, vol. 28, no. 5, p. 1249-1256, DOI:10.1108/03321640910969494.

[8] Encica, L., Echeverria, D., Lomonova, E.A., Vandenput, A.J.A., Hemker, P.W., Lahaye, D. (2007). Efficient optimal design of electromagnetic actuators using space mapping. Structural and Multidisciplinary Optimization, vol. 33, no. 6, p. 481-491, DOI:10.1007/ s00158-006-0054-6.
[9] Encica, L., Paulides, J.J.H., Lomonova, E.A., Vandenput, A.J.A. (2008). Aggressive output spacemapping optimization for electromagnetic actuators. IEEE Transactions on Magnetics, vol. 44, no. 6, p. 1106-1109, DOI:10.1109/TMAG.2007.916224.

[10] Markovic, M., Jufer, M., Perriard, Y. (2004). Analyzing an electromechanical actuator by Schwarz-Christoffel mapping. IEEE Transactions on Magnetics, vol. 40, no. 4, p. 1858-1863, DOI:10.1109/TMAG.2004.828920.

[11] Markovic, M., Jufer, M., Perriard, Y. (2008). Analytical force determination in an electromagnetic actuator. IEEE Transactions on Magnetics, vol. 44, no. 9, p. 2181-2185, DOI:10.1109/TMAG.2008.888573.

[12] Chung, M.J., Gweon, D.G. (2003). Optimal design and development of electromagnetic linear actuator for mass flow controller. KSME International Journal, vol. 17, no. 1, p. 40-47.

[13] Cai, B.P., Liu, Y.H., Tian, X.J., Wang, Z.L., Wang, F., Li, H., Ji, R.J. (2011). Optimization of Submersible Solenoid Valves for Subsea Blowout Preventers. IEEE Transactions on Magnetics, vol. 47, no. 2, p. 451-457, DOI:10.1109/TMAG.2010.2100825.

[14] Batdorff, M.A., Lumkes, J.H. (2009). High-fidelity magnetic equivalent circuit model for an axisymmetric electromagnetic actuator. IEEE Transactions on Magnetics, vol. 45, no. 8, p. 3064-3072, DOI:10.1109/ TMAG.2009.2017531.

[15] Topcu, E.E., Kamis, Z., Yuksel, I. (2008). Simplified numerical solution of electromechanical systems by look-up tables. Mechatronics, vol. 18, no. 10, p. 559565, DOI:10.1016/j.mechatronics.2008.05.006.

[16] Sefkat, G. (2009). The design optimization of the electromechanical actuator. Structural and Multidisciplinary Optimization, vol. 37, no. 6, p. 635644, DOI:10.1007/s00158-008-0254-3.

[17] Zhao, J.F., Seethaler, R.J. (2010). Compensating combustion forces for automotive electromagnetic valves. Mechatronics, vol. 20, no. 4, p. 433-441, DOI:10.1016/j.mechatronics.2010.03.003.

[18] Lovisolo, A., Roccato, P.E., Zucca, M. (2008). Analysis of a magnetostrictive actuator equipped for the electromagnetic and mechanical dynamic characterization. Journal of Magnetism and Magnetic Materials, vol. 320, no. 10, p. e915-e919, DOI:10.1016/j.jmmm.2008.04.065.

[19] Cai, B.P., Liu, Y.H., Ren, C.K., Abulimiti, A., Tian, X.J., Zhang, Y.Z. (2012). Probabilistic Thermal and Electromagnetic Analyses of Subsea Solenoid Valves for Subsea Blowout Preventers. Strojniški vestnik Journal of Mechanical Engineering, vol. 58, no. 11, p. 665-672, DOI:10.5545/sv-jme.2012.681.

[20] Cai, B.P., Liu, Y.H., Tian, X.J., Wang, F., Li, H., Ji, R. (2010). An experimental study of crevice corrosion behaviour of 316L stainless steel in artificial seawater. Corrosion Science, vol. 52, p. 3235-3242, DOI:10.1016/j.corsci.2010.05.040.

[21] Cai, B.P., Liu, Y.H., Tian, X.J., Li, H., Ji, R., Wang, F., Zhang, Y.Z. (2011). Susceptibility of 316L stainless 
steel to crevice corrosion in submersible solenoid valve. Materials and Corrosion, vol. 62, no. 8, p. 753759, DOI:10.1002/maco.201005917.

[22] Tanaka, S., Ueda, K., Mitamura, N., Oohori, M. (2006). The Development of an austenitic stainless steel bearing with high corrosion resistance and high nonmagnetic property. Journal of ASTM International, vol. 3, no. 9, p. 1790-2013, DOI:10.1520/JAI100424.

[23] Angadi, S.V., Jackson, R.L., Choe, S.Y., Flower, G.T., Suhling, J.C., Chang, Y.K., Ham, J.K. (2009). Reliability and life study of hydraulic solenoid valve. Part 1 A multi-physics finite element model. Engineering Failure Analysis, vol. 16, no. 3, p. 974887, DOI: 10.1016/j.engfailanal.2008.08.011.

[24] Oriol, G.B., Campanile, L.F. (2010). Design Rules for Actuator in Active Mechanical Systems, 1st ed., Springer, London.
[25] Oriol, G.B., Samuel, G.A., Antoni, S.A., Daniel, M.M., Campanile, L.F. (2007). Linear electromagnetic actuator modeling for optimization of mechatronic and adaptronic systems. Mechatronics, vol. 17, no. 2-3, p. 153-163, DOI:10.1016/j.mechatronics.2006.07.002.

[26] Haskara, I., Kokotovic, V.V., Mianzo, L.A. (2004). Control of an electro-mechanical valve actuator for a camless engine. International Journal of Robust and Nonlinear Control, vol. 14, no. 6, p. 561-579, DOI:10.1002/rnc.903.

[27] Topcu, E.E., Yuksel, I., Kamis, Z. (2006). Development of electro-pneumatic fast switching valve and investigation of its characteristics. Mechatronics, vol. 16, no. 6, p. 365-378, DOI:10.1016/j. mechatronics.2006.01.005. 